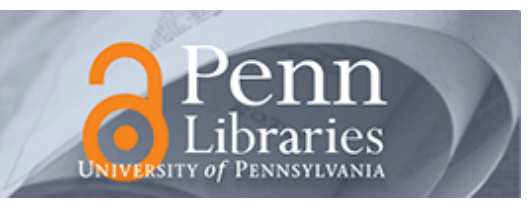

University of Pennsylvania

ScholarlyCommons

June 2000

\title{
A Finite Element Model of the Breast for Predicting Mechanical Deformations during Biopsy Procedures
}

\author{
Fred S. Azar \\ University of Pennsylvania \\ Dimitris N. Metaxas \\ University of Pennsylvania \\ Mitchell D. Schnall \\ University of Pennsylvania, Schnall@oasis.rad.upenn.edu
}

Follow this and additional works at: https://repository.upenn.edu/hms

\section{Recommended Citation}

Azar, F. S., Metaxas, D. N., \& Schnall, M. D. (2000). A Finite Element Model of the Breast for Predicting Mechanical Deformations during Biopsy Procedures. Retrieved from https://repository.upenn.edu/hms/44

Copyright 2000 IEEE. Reprinted from Proceedings of the IEEE Workshop on Mathematical Methods in Biomedical Image Analysis, June 2000, pages 38-45.

Publisher URL: http://dx.doi.org/10.1109/MMBIA.2000.852358

This material is posted here with permission of the IEEE. Such permission of the IEEE does not in any way imply IEEE endorsement of any of the University of Pennsylvania's products or services. Internal or personal use of this material is permitted. However, permission to reprint/republish this material for advertising or promotional purposes or for creating new collective works for resale or redistribution must be obtained from the IEEE by writing to pubs-permissions@ieee.org. By choosing to view this document, you agree to all provisions of the copyright laws protecting it.

This paper is posted at ScholarlyCommons. https://repository.upenn.edu/hms/44

For more information, please contact repository@pobox.upenn.edu. 


\title{
A Finite Element Model of the Breast for Predicting Mechanical Deformations during Biopsy Procedures
}

\author{
Abstract \\ Currently, High Field (1.5T) Superconducting MR image-guided needle breast procedures allow the \\ physician only to calculate approximately the location and extent of a cancerous tumor in the \\ compressed patient breast before inserting the needle. It can then become relatively uncertain that the \\ tissue specimen removed during the biopsy actually belongs to the lesion of interest. A new method for \\ guiding clinical breast biopsy is presented, based on a deformable finite element model of the breast. The \\ geometry of the model is constructed from MR data, and its mechanical properties are modeled using a \\ non-linear material model. This method allows imaging the breast without compression before the \\ procedure, then compressing the breast and using the finite element model to predict the tumor's position \\ during the procedure.

\section{Comments} \\ Copyright 2000 IEEE. Reprinted from Proceedings of the IEEE Workshop on Mathematical Methods in \\ Biomedical Image Analysis, June 2000, pages 38-45. \\ Publisher URL: http://dx.doi.org/10.1109/MMBIA.2000.852358 \\ This material is posted here with permission of the IEEE. Such permission of the IEEE does not in any way \\ imply IEEE endorsement of any of the University of Pennsylvania's products or services. Internal or \\ personal use of this material is permitted. However, permission to reprint/republish this material for \\ advertising or promotional purposes or for creating new collective works for resale or redistribution must \\ be obtained from the IEEE by writing to pubs-permissions@ieee.org. By choosing to view this document, \\ you agree to all provisions of the copyright laws protecting it.
}




\section{A Finite Element Model of the Breast for Predicting Mechanical Deformations during Biopsy Procedures}

\author{
Fred S. Azar \\ U. of Pennsylvania \\ Dept. of BioEngineering \\ fredazar@seas.upenn.edu
}

\author{
Dimitris N. Metaxas \\ U. of Pennsylvania \\ Dept. of Computer Science \\ dnm@graphics.cis.upenn.edu
}

\author{
Mitchell D. Schnall \\ U. of Penn Medical Center \\ Dept. of Radiology \\ schnall@oasis.rad.upenn.edu
}

\begin{abstract}
Currently, High Field (1.5T) Superconducting MR image-guided needle breast procedures allow the physician only to calculate approximately the location and extent of a cancerous tumor in the compressed patient breast before inserting the needle. It can then become relatively uncertain that the tissue specimen removed during the biopsy actually belongs to the lesion of interest. A new method for guiding clinical breast biopsy is presented, based on a deformable finite element model of the breast. The geometry of the model is constructed from MR data, and its mechanical properties are modeled using a non-linear material model. This method allows imaging the breast without compression before the procedure, then compressing the breast and using the finite element model to predict the tumor's position during the procedure.
\end{abstract}

\section{Introduction}

It is impossible today to do continuous breast imaging using High Field (1.5T) Superconducting Magnetic Resonance Imaging. MR image-guided needle procedures such as needle localizations require that the patient lie in a prone position with the breast gently compressed between medial and lateral plates. The lateral plate contains a grid of approximately 1000 holes placed at 5-mm intervals, which guide the needle in a plane parallel to the tabletop. The location of the cancerous lesion is calculated from the contrast-enhanced static breast MR images. The correct hole in the plate is identified and a needle is inserted through that hole into the breast with a pre-calculated depth. An additional problem that arises is that the use of compression plates during imaging after a contrast-agent injection [1] may critically change the enhancement characteristics of the tumor, making the tracking of its boundaries very difficult. The above limitations coupled with the deformable structure of the breast makes needle procedures very sensitive to the initial placement of the needle; it thus becomes relatively uncertain that the tissue specimen removed during the biopsy procedure actually belongs to the lesion of interest.
A new method for tracking the position of a hard inclusion in soft tissue is presented, based on a deformable finite element model (FEM) of the breast. The geometry of the model is constructed from MR data, and its mechanical properties are based on a non-linear material model. This method allows imaging the breast without compression before the procedure, then compressing the breast and using the finite element model to predict the tumor's position during the procedure [2].

A patient's left breast was imaged uncompressed and then imaged under two different compression amounts $(12 \%$, and $26 \%)$. The corresponding deformable model was built, and underwent virtual compressions similar to the real compressions. We tracked the motion of a small cyst inside the patient's breast, and used the deformable model to predict the cyst's position in the compressed breast. We also tracked the motion of two vitamin E pills attached to the surface of the patient's breast.

The results suggest that it is possible to create a deformable model of the breast based on the use of finite elements with non-linear material properties capable of modeling the deformation of the breast in a reasonably short amount of time (< one half-hour).

\section{Background}

\subsection{Physiology of the breast}

Figure 1 shows the major structures of a typical mature pre-menopausal breast. The breast is an inhomogeneous structure containing many layers of many different kinds of tissue. However the two predominant types of tissue within the breast are fat and normal glandular tissue, which supports lactation. The superficial layer (fascia) is separated from the skin by 0.5 to $2.5 \mathrm{~cm}$ of subcutaneous fat. Tentacle-like prolongations of fibrous tissue extend in all directions from this fascia to the skin; these are called Cooper's ligaments. In the adult mammary gland, there are 15 to 20 irregular lobes, converging to the nipple through ducts 2 to $4.5 \mathrm{~mm}$ in diameter [3]. These ducts are immediately surrounded by dense connective tissue, which acts as a supporting framework. Carcinomas in affected breasts are usually accompanied by local changes in material properties, due to increased stiffness in the lesion, as well as its shape and size [4]. There are several 
types of breast cancer, however the most common are ductal carcinoma (which begins in the lining of the milk ducts of the breast), and lobular carcinoma (which begins in the lobules where breast milk is produced) [5].

\subsection{Problems encountered by the MR imaging- guided localization techniques}

The MR imaging-guided localization techniques encounter the following problems:

- The appearance, size and shape of the potential cancer lesion greatly depend on dynamics of the contrast-enhancing agent. As explained above, the lesion may clearly appear only in the two minutes following the contrast agent injection, then the signal intensity may vary arbitrarily, and it is quite possible that the apparent boundaries of the lesion may change dramatically.

- $\quad$ The use of compression plates when imaging the breast after injection of the contrast-enhancing agent may change the enhancement characteristics of the tumor and could very well make the lesion disappear.

- $\quad$ The deformable nature of the breast makes it very difficult to stabilize, and both external movements (due to the patient), and internal deformations occur as the needle is inserted.

- $\quad$ The compression plates could compress the breast to a degree that makes it virtually immobile, however this would cause a high level of discomfort to the patient in addition to the possible anxiety, in addition to the possible altered enhancement characteristics of the tumor as mentioned above.

- $\quad$ Furthermore, the guidance techniques all use static MR images for localizing the lesions (pre and then postcontrast). It is impossible today to do continuous breast imaging using high field superconducting magnetic resonance imaging. Therefore live imaging can't be maintained throughout the entire procedure.
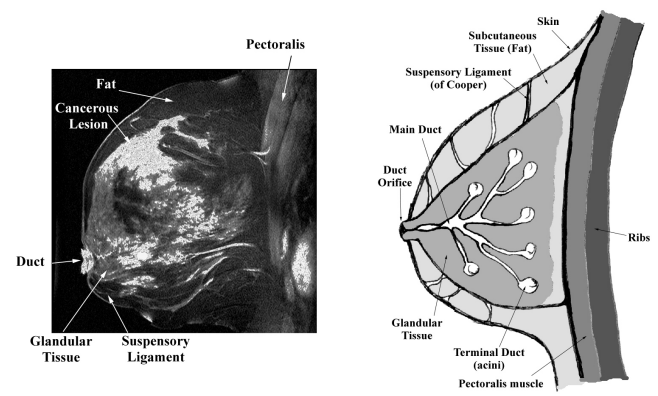

Figure 1. Glandular and supporting framework of the breast

The above limitations coupled with the deformable structure of the breast makes needle procedures very sensitive to the initial placement of the needle. It thus becomes relatively uncertain that the tissue specimen removed during the biopsy procedure actually belongs to the lesion of interest, due to the added difficulty of accurately locating the tumor's boundaries inside the breast.

We present a new method for guiding clinical breast biopsy, based on the use of an accurate virtual deformable breast model of the patient whose geometry is constructed from MR data. The elastic properties of the deformable model are based on the use of finite elements with nonlinear material properties, capable of modeling the deformation of the breast. This method will allow imaging of the breast without any compression before a needle procedure, then compress the breast, and its virtual finite element model (by applying the same pressure to both). It is also important that the model simulation runs reasonably fast enough to be useful in a clinical environment.

A finite element model of the breast will be a very flexible tool for many applications including those mentioned above, and also for diagnosis, measurements, surgery planning, simulations of deformation due to inserting a needle, and further away, virtual surgery, and even tele-surgery.

\subsection{Background on deformable models}

Finite element modeling has been used in a very large number of fields. However it is only recently that deformable models have been used to simulate deformations in soft tissue. Physical models are among the first to be used. Among these physical models, elastic (linear and visco-elastic) models have been extensively described in the literature [6-8]. The most widely used representations for deformable volumes are parametric models with B-spline representation [9]. Other possible models are mass-spring models [10-12], and implicit surfaces [13]. The mass-spring methods have been used most of the time for surgery simulation due to their simplicity of implementation and their lower computational complexity [14-16].

Finite element models are less widely used due to the difficulty of their implementation and their larger computing time. There are many powerful commercial F.E.M. packages that allow complex simulations of deformation such as ABAQUS (HKS, Rhode Island); Breast tissue is relatively complex, and consists of layers of different tissues interlaced with ligaments and fascias. Very complex models would be needed to model these objects realistically. However the complexity of the model and the required computational time (which can extend to several days on an SGI Indigo2 workstation) would prohibit these models from being useful clinically. Few models of the breast have actually been implemented using the commercial software packages, and have mostly involved phantom studies $[2,17,18]$.

In order to develop a model for deformation of the breast, we must decide on a geometric description of the breast, a mathematical model of the elastic deformation, 
and a solution algorithm that is both fast and yields a reasonably realistic result. For that reason, what matters most is that the breast deformation results be realistic and available in a clinically useful time (less than thirty minutes), and that the model be robust and show a consistent and predictable behavior.

In our approach, we strive to integrate the requirements for a realistic simulation of deformation, and the reasonably fast time modeling which is a necessity if the model is to be used in a clinical environment: by incorporating the geometric definition of the breast model into the physics-based framework developed in [19, 20], we create a deformable breast model, capable of reasonably predicting the internal deformations of a real patient breast after plate compression.

\section{Methods}

\subsection{Construction of the finite element model (F.E.M) of the breast}

Because of the high variability of breast shapes and the deformation of the breast when compressed in order to be imaged, the model devised closely follows the contours of the patient breast.

3.1.1. MRI data. The patient data is a set of parallel $2 \mathrm{D}$ spoiled gradient echo MR axial slices of the breast: they are called 'axial localizers', and are actually imaged before every examination to the breast. This makes the availability of such data very high and no additional scans are needed, to the benefit of the patient. Usually, an axial T1-weighted spin echo sequence is performed with a repetition time of $500 \mathrm{msec}$, and an echo time of $12 \mathrm{msec}$, with a $12-16 \mathrm{~cm}$ field of view, a 3-mm thick section, and a 256x256 matrix. The acquisition ensures a 3D visualization of the patient's breast.

\subsubsection{Segmentation and classification of the MR data.} First the MR image 3D set is converted into a set of axial slices (if the original data is not axial) through automatic resampling of the data using a software package such as Scion Image (Scion Corp., Maryland).

The MR images are loaded to a program, which enables segmentation through contours. The contours of the breast boundary are created semi-automatically using Scion Image and Photoshop (Adobe Corp.), and saved to a file for subsequent use. At the same time each MR image is manually segmented into parenchyma, fat and/or lesion tissue, and the results are also stored in a file (each tissue type is assigned a specific gray level value).

When displayed in 3D, the breast contours appear as in Figure 2 (here the breast is being compressed between two parallel plates, giving it the shape in Figure 2).
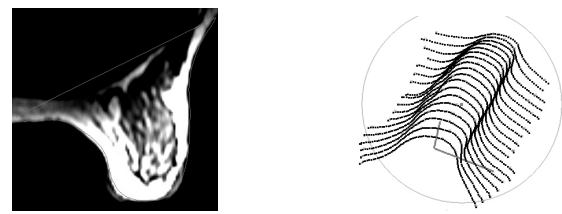

Figure 2. Axial MR slice of breast from axial localizer (left) and breast contours (right)

3.1.3. 3D mesh domain creation. BreastView, a custom-written program in $C$, takes as input the set of breast contours and the segmentation results. Running on an SGI workstation, it generates the 3D computational domain (mesh) of the breast in a few seconds, allowing to scale the volume elements to any size.

The program can also generate a finite element model (FEM) file readable by FEM software ABAQUS. This file contains the definition of the volume elements, as well as the boundary conditions, and the different material properties. Two types of 3D volume elements are used in the model (Figure 3):

- $\quad$ Solid 8-node trilinear isoparametric elements: these elements are used to model all breast tissues except for the skin, and are assigned non-linear material properties,

- 3-node triangular isoparametric elements: these elements are used to model the skin, and are assigned non-linear elastic properties in the plane of their triangular surface, and no axial stiffness.
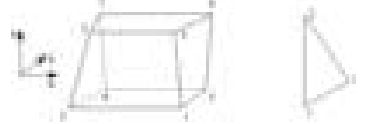

Figure 3. Elements used to build the deformable model: 8-node element (left), 3-node triangle (right)

BreastView allows the mesh to be as dense as needed. These volume elements are well suited for the purpose of the model: a physician using the model to predict the internal motion of a small tumor for example, could include the tumor in one volume element (by correctly scaling the mesh density) which would be assigned its own mechanical characteristics. Figure 4 shows a contour and the 2D mesh generated in it.

In order to create the $3 \mathrm{D}$ mesh, we first find the principal direction $d$ (direction of the $y$-axis, Figure 4), from the chest wall to the nipple. Any line with direction $d$ in the plane of the contour, which intersects the contour, intersects it twice. This direction is that of the line orthogonal to the line which passes through the two endpoints (the principal direction method using the eigenvectors of the contour set of points did not yield the best direction $d$ ). The center of gravity $G$ of the contour set is calculated. The 3D mesh can now be easily generated following the $U$ (along $x$ ) and $V$ (along $y$ ) resolutions desired, in the orthogonal $(x, y)$ basis centered at $G$. This algorithm ensures that we have the same 
number of points on every $V$-line, and the same number of $V$-lines on every slice.

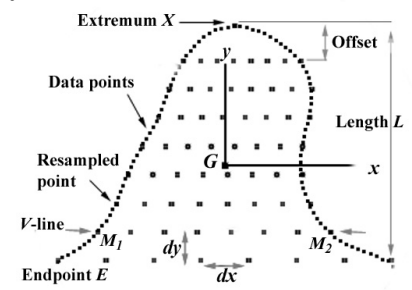

Figure 4. Slice contour, and 2D mesh generation

3.1.4. Material properties assignment. Once the $3 \mathrm{D}$ mesh is created, each element is assigned a material property corresponding to that of fat, parenchyma or cancerous tissue. In order to determine what type of material property to assign to any one $3 \mathrm{D}$ element, an algorithm makes use of the manually segmented breast MR data. The algorithm calculates what percentage of fat, parenchyma and cancerous tissue lie within the element, by 'counting' the corresponding number of voxels which lie in the element. The material property that corresponds to the highest percentage is the one assigned to the element.

3.1.5. Initial and boundary conditions. Usually, two compression plates are used to compress the breast. Here we make the following assumptions, which correspond to the actual breast compression setup:

- $\quad$ The plates are parallel to the $y$-axis

- $\quad$ The plates move in the direction of the $x$-axis, towards the breast, in a way that displacements can be considered static

- The total compression distance $D_{\text {tot }}$ for a plate is from its first point of contact with the breast to its final resting position.

Once the physical parameters are known, such as the plate length $L_{p}$, the distance from its tip to the patient's rib cage $D_{t}$, and the total compression distance $D_{t o t}$, we translate those parameters into prescribed displacements to all individual model surface nodes, which come in contact with the plate.

The total compression distance is translated into $m_{\text {tot }}$ number of small displacement iterations. A simple collision detection algorithm determines which node $i$ comes in contact with the plate at each displacement iteration. Each of these nodes is assigned a prescribed displacement increment $d_{n i}$ repeated in every iteration for which the plate is in contact with the node. Therefore the number of iterations for every surface node $i$ varies and is denoted $m_{n i}$.

All nodes in the first two planes at the base of the breast model are fixed. This provides the support structure and fixes the breast model in space.

\subsection{Applying large deformations to the F.E.M. of the breast}

3.2.1. Modeling large deformations. Compressing a breast using compression plates results in a large compression of the breast, meaning that the total compression distance between the two plates decreases by more than $10 \%$. In order to model such large deformations, we divide the large deformation compression into a number of much smaller displacement steps (as described in the previous section). For every displacement step, we make use of small strain theory. Strain is calculated using Cauchy's infinitesimal strain tensor formula (given in the unabridged notation [21]):

$$
\begin{array}{ccc}
\varepsilon_{x x}=\frac{\partial u}{\partial x}, & \varepsilon_{y y}=\frac{\partial v}{\partial y}, & \varepsilon_{z z}=\frac{\partial w}{\partial z} \\
\varepsilon_{x y, y x}=\frac{1}{2}\left(\frac{\partial u}{\partial y}+\frac{\partial v}{\partial x}\right), & \varepsilon_{x z, z x}=\frac{1}{2}\left(\frac{\partial u}{\partial z}+\frac{\partial w}{\partial x}\right), & \varepsilon_{y z, z y}=\frac{1}{2}\left(\frac{\partial v}{\partial z}+\frac{\partial w}{\partial y}\right) .
\end{array}
$$

where $u=u(x, y, z), \quad v=v(x, y, z), \quad w=w(x, y, z)$ are the displacement fields in the $x, y$ and $z$ directions respectively, from one small displacement iteration to another.

3.2.2. Modeling non-linear material properties. Most biological tissues display both a viscous (velocity dependent) and elastic response, however since we are only interested in slow displacements, the great majority of the forces developed can be attributed purely to the elastic response.

All tissues involved in the breast can be considered:

- Isotropic [22, 23],

- Homogeneous [24, 25],

- Incompressible [26],

- To have non-linear elastic properties [27, 28].

With these assumptions, it is possible to define the mechanical behavior of breast tissue using a single elastic modulus $E_{n}$, which is a function of strain $\varepsilon_{n}$ for tissue type $n\left(\sigma_{n}\right.$ is the stress):

$$
E_{n}=\frac{\partial \sigma_{n}}{\partial \varepsilon_{n}}=f\left(\varepsilon_{n}\right)
$$

This non-linear relationship is calculated for every tissue type from uniaxial stress-strain experiments using tissue samples. The experimental curves are fit to a material model, which can be characterized using a small number of parameters.

In order to model the non-linear mechanical behavior in every element, the following steps are followed, after every small deformation increment:

- We calculate the Lagrangian strain tensor, $\boldsymbol{E}$, at the center of the element. $\boldsymbol{E}$ is a measure of the deformation of a point in the model with respect to its initial position (a detailed description can be found in [29]):

$\boldsymbol{E}=\frac{1}{2}\left(\boldsymbol{F}^{T} \boldsymbol{F}-\boldsymbol{I}\right)$

$\boldsymbol{F}$ is the 3x3-deformation gradient tensor, and its components can be written as: 


$$
F_{p q}=\frac{\partial x_{p}}{\partial X_{q}}
$$

where $x_{p}$ is one of the three components of $\boldsymbol{x}$, the final position vector, and $X_{q}$ is one of the three components of $\boldsymbol{X}$ the initial position vector. In our case, the final position of an element center point is after a given deformation increment, and the initial center point is before the first deformation increment. $\boldsymbol{I}$ is the identity matrix.

- We calculate the maximum principal strain component, $e_{\max }$, which is given by the largest eigenvalue of the strain tensor $\boldsymbol{E}$.

- Using the experimental stress-strain curve for the particular tissue type, the element is assigned a Young's modulus value corresponding to the slope of the curve at the strain measure $e_{\max }: E_{n}=f\left(e_{\max }\right)$

Therefore after every deformation increment, the element's stiffness value is updated to model the material's non-linear behavior. By doing so, we model a continuously differentiable stress-strain curve (in the limits of our deformations) as a continuous step-wise linear curve.

3.2.3. Model dynamics. The governing Lagrange equations of motion are second order differential equations given as follows $[19,20]$ :

$$
\boldsymbol{M} \ddot{\boldsymbol{q}}+\boldsymbol{D} \dot{\boldsymbol{q}}+\boldsymbol{K} \boldsymbol{q}=\boldsymbol{g}_{q}+\boldsymbol{f}_{\boldsymbol{q}}
$$

where $\boldsymbol{M}, \boldsymbol{D}$ and $\boldsymbol{K}$ are the mass, damping and stiffness matrices respectively. The vector $\boldsymbol{q}$ contains the displacement degrees of freedom, $\boldsymbol{g}_{q}$ are the inertial forces arising from the dynamic coupling between the local and global degrees of freedom, and $f_{q}$ are the generalized external forces associated with the model's degrees of freedom.

In our case, given the nature of the problem, and the fact that we consider static deformations only, we neglect the inertial term, we assume zero acceleration, and we do not impose any physical damping. Therefore the breast model is deformed using the following reduced form of Lagrange's equation of motion:

$$
\dot{q}+K q=f_{q}
$$

where the vector $\boldsymbol{q}$ contains the displacement degrees of freedom and the vector $f_{q}$ contains the total external forces due to body forces such as gravity. Stiffness (represented by the $\boldsymbol{K}$ matrix) can also be viewed as an internal resistance which results in a force equal to $\boldsymbol{K q}$. Lagrange's equation of motion can then be written for each and every node $i$ in the model as [30] :

$$
\dot{q}_{i}=f_{i, \text { total }}=f_{i, \text { internal }}+f_{i, \text { external }}
$$

where $\boldsymbol{q}_{\boldsymbol{i}}$ is the 3D nodal displacement, $\boldsymbol{f}_{\text {i,internal }}=[-\boldsymbol{K} \boldsymbol{q}]_{i}$ is an internal stiffness force, and $\boldsymbol{f}_{i, \text { external }}$ is an external force combining the body forces. At every displacement iteration, we integrate equation (7) using the Euler technique [31,32], with a step size $h$.

3.2.4. Internal forces due to stiffness. The degrees of freedom of the model are the 3D displacements of the finite element nodes. In our formulation, stiffness is represented as an internal force $f_{\text {i,intermal }}$. The element force vector $f_{e}$ contains the forces on the element nodes. It is calculated for each element as:

$f_{e}=K d_{e}$

where $\boldsymbol{d}_{e}$ contains the nodal displacements. The stiffness matrix, $\boldsymbol{K}$, incorporates the geometry and material properties of the element and is computed from:

$\boldsymbol{K}=\iiint \boldsymbol{B}^{T} \boldsymbol{D} \boldsymbol{B} d V$

The matrix $\boldsymbol{D}$ contains the material property information, (which includes the value of the material's elastic modulus $E_{n}\left(\varepsilon_{n}\right)$ evaluated as described previously) and relates nodal stresses $\sigma$, to nodal strains $\varepsilon$ :

$\sigma=D \varepsilon$

The matrix $\boldsymbol{B}$ relates nodal strains to displacements, and incorporates Cauchy's infinitesimal strain tensor formulation (see equation (1)):

$\varepsilon=\boldsymbol{B} \boldsymbol{d}_{e}$

Once the element forces are calculated, each element contributes to the total internal nodal force $f_{\text {i,internal }}$ for each of its nodes. A detailed derivation of the finite element formulation for calculating the stiffness matrix, $\boldsymbol{K}$, is presented in [33].

\section{Experimental design and methods}

\subsection{Mechanical measurements of human breast tissue}

Few studies have been made on determining the mechanical properties of tissue in the breast, however average values of Young's modulus have been calculated for fat, glandular tissue, and cancer tissue [23, 25, 34]. Since we need non-linear stress-strain curves describing the mechanical behavior of breast tissue, we will use the mechanical properties of breast tissue determined by Wellman et al. [35].

Exponential curves (which have been used in the past for several different tissue types [26]) are used to describe the stress-strain properties of breast tissue, following experimental stress-strain curves obtained from uniaxial loading of breast tissue [35, 36]:

$E_{n}=\frac{\partial \sigma_{n}}{\partial \varepsilon_{n}}=b \cdot e^{m \varepsilon_{n}}$

where $\sigma_{n}$ and $\varepsilon_{n}$ are the nominal stress and strain respectively for tissue type $n . b$ and $m$ are the fit parameters determined experimentally for every tissue type:

- $b_{\text {glandular }}=15100 \mathrm{~N} / \mathrm{m}^{2}$

- $m_{\text {glandular }}=10.0$ (within 1 standard deviation) 
- $b_{\text {fat }}=4460 \mathrm{~N} / \mathrm{m}^{2}$

- $m_{\text {fat }}=7.4$ (within 1 standard deviation)

\subsection{Breast compression patient study}

A patient's left breast was used for the patient study. We found a small cyst inside the breast, which was used as the inner landmark for tracking displacements inside the breast after compression.

4.2.1. Patient breast MR data acquisition. The breast was imaged using a 1.5 Tesla machine Signa Horizon Echospeed (GEMS, Milwaukee). 3D image sets were obtained under the different plate pressure conditions. The entire breast was imaged medially to laterally, from the visible rib cage to the nipple. The MR acquisition sequence was a 3D fast SPGR (T1 weighted), with a TR of $11.3 \mathrm{~ms}$, a TE of $4.2 \mathrm{~ms}$ and a 25 degrees flip angle. We used a phased array multi coil. 124 slices each with a 0.9 $\mathrm{mm}$ thickness were acquired sagitally (left to right). Each slice had a field of view of $230 \mathrm{~mm} \times 230 \mathrm{~mm}(256 \times 256$ pixels). This amounts to having cubic voxels with $0.9 \mathrm{~mm}$ sides.

4.2.2. Initial and boundary conditions. The breast was imaged first uncompressed, then imaged with two different plate compression amounts. The breast compression device, made by GEMS, stabilized the patient's breast well enough to minimize motion artifacts between image sets. The compression plates were able to slide perfectly in the sagittal direction and were locked in position when the desired compression was achieved. The displacements can therefore be considered static.

In order to help track the movement of the breast, we taped to the surface of the breast two vitamin E pills (one towards the superior part, the other one towards the inferior part) which appear as bright spots on the MR images, these serve as landmarks. The right plate (on the breast's medial side) was kept immobile, while the left plate (on the lateral side) was moved to compress the breast.

Table 1. Plate to Plate Distances Before and After Compression

\begin{tabular}{|l|c|c|}
\hline & $\begin{array}{c}\text { PLATE TO PLATE } \\
\text { DISTANCE (MM) }\end{array}$ & $\begin{array}{c}\text { COMPRESSION } \\
\text { AMOUNT (MM) }\end{array}$ \\
\hline UNCOMPRESSED & 64.8 & 0.0 \\
\hline COMPRESSION 1 & 56.7 & 8.1 \\
\hline COMPRESSION 2 & 47.7 & 17.1 \\
\hline
\end{tabular}

4.2.3. Patient breast deformable model. A model of the patient breast was constructed from the given MR data, the boundary conditions, and the applied displacements, using BreastView. The different breast tissues inside the breast were modeled (glandular and fatty).
For the breast compression simulation, the skin was not modeled since the skin's stiffness is mainly responsible for counteracting the effects of the gravitational force when the patient lies prone. Furthermore since the applied compression is mainly parallel to the skin's surface, we neglected the mechanical effects of the skin when doing plate compression.

The breast data was segmented using an image analysis software such as Scion Image and Photoshop, starting at the intersection of the outer side of one compression plate and the breast, all the way to the other plate.

The deformable model of the breast was built using every other slice out of the experimental data set, and discarding the outer slices, which did not contain useful information. A total of 58 slices were used. The breast model consists therefore of 58 slices (each slice in the $x y$ plane) stacked up along the $z$-axis. We chose to have 8 nodes in the $x$-direction, and 8 nodes in the $y$-direction in every slice, in order to keep the simulation running time under a 1/2 hour. The model contains 3712 nodes, and 2793 finite elements.

We did two sets of experiments:

a) We applied the full deformation amount using only the right compression plate, and leaving the left compression plate immobile.

b) We used both plates to compress the deformable model, each plate compressing half of the full amount.

In both cases we used an integration step size $h$ of $10^{-5}$, the simulation took about a half-hour on a Silicon Graphics Octane workstation.

\section{Results and discussion}

\subsection{Experimental results}

Table 3 shows the displacement vectors of the cyst inside the breast, and the two vitamin $\mathrm{E}$ pills for each of the two plate compressions. The displacement measurements (in $\mathrm{mm}$ ) were made using Scion Image software. The edge points of the vitamin E pills closest to the skin were used in the calculations.

\subsection{Deformable model results}

5.2.1. Material properties modifications. We modeled the two breast compressions using the material properties as described above. However, the values used for fatty tissue caused the 'fat' elements to collapse on themselves after a few displacement iterations. This would mean in reality that the fat is squeezed out of its location as the breast is being compressed, which would make sense since fat is almost liquid at body temperature. Now in accordance with the experimental results of compressed breasts scanned to date, it is clear that the fatty tissue is not squeezed out, and when compressed, it actually remains in its location. Although the experimental values for the mechanical properties of fat are accurate, we must remember that the measurements of those properties were 
made ex-vivo, and did not take into account the supporting structure of fibers that exists in the breast. We hypothesize that it is this structure including Cooper's ligaments that compartmentalizes the fatty tissue in the breast, and prevents it from being squeezed out of its location. Therefore as the fatty tissue is being compressed, and prevented from being squeezed out, the local pressure should increase, and should lead to an increase in the apparent stiffness value of fatty tissue.

We tested this hypothesis by changing the Young's modulus $E_{\text {fat }}$ formula for the fatty tissue, to a simpler linear equation given by:

$$
E_{\text {fat }}\left(\varepsilon_{\text {fat }}\right)=\left\{\left(\begin{array}{cc}
\left(\frac{E_{\text {gland_avg }}-b_{\text {fat }}}{\varepsilon_{\text {limit }}}\right) \varepsilon_{\text {fat }}+b_{\text {fat }} \text { if } \varepsilon_{\text {fat }}<\varepsilon_{\text {limit }} \\
E_{\text {gland_avg }} & \text { if } \varepsilon_{\text {fat }} \geq \varepsilon_{\text {limit }}
\end{array}\right\}\right.
$$

where $E_{\text {gland avg }}$ is an average stiffness value for glandular tissue and is the calculated Young's modulus of glandular tissue for a limit $\varepsilon_{\text {limit }}=25 \%$ strain: $E_{\text {gland_avg }}=184 \mathrm{kN} / \mathrm{m}^{2}$. Therefore the stiffness of fatty tissue was made to increase linearly up to the stiffness value of glandular tissue. The value for $\varepsilon_{\text {limit }}$ was determined after iterating through five different strain values.

Table 2. MR Slices Containing the Landmarks, for the Uncompressed Case
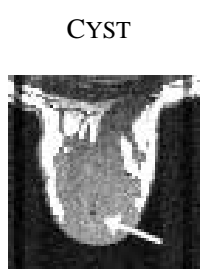
VITAMIN E PILL (INFERIOR)

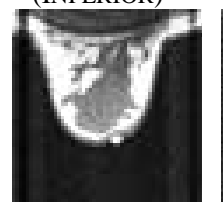

VITAMIN E PILL (SUPERIOR)
UNCOMPRESSED<smiles>[3H][13CH3]</smiles>

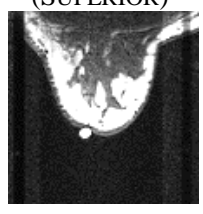

5.2.2. Compression results. Table 4 and Table 5 show the relevant model meshes in the $x y$-plane, superimposed on the MR data. The landmarks' initial positions were recorded in the undeformed model, and their final position in the compressed model was interpolated from the finite elements' shape functions.

The errors in displacement between the modeled and the experimental data are shown below in Table 6. The boundary-fit error is an average in the x-direction of the error between the boundary nodes of the model, and the surface of the breast for the three slices involved.

The imaging error is $0.9 \mathrm{~mm}$ in the $x$, and $y$ direction (the plane of the imaged slices), and $1.8 \mathrm{~mm}$ in the $z$ direction. The results show that the one-plate virtual compression of the deformable model performed better and with less errors in displacements than the two-plate virtual compression. However the boundary-fit error was much lower in both compressions in the two-plate virtual compression simulation.

Table 3. Displacement Vectors $(\mathrm{mm})$ of the Landmarks Obtained Experimentally (Compressed-Uncompressed)

\begin{tabular}{|c|c|c|c|c|c|c|c|c|c|}
\hline \multirow{2}{*}{ 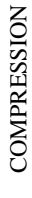 } & \multicolumn{3}{|c|}{$\begin{array}{l}\text { VITAMIN E PILL } \\
\text { (SUPERIOR) }\end{array}$} & \multicolumn{3}{|c|}{$\begin{array}{l}\text { VITAMIN E PILL } \\
\text { (INFERIOR) }\end{array}$} & \multicolumn{3}{|c|}{ SMALL CYST } \\
\hline & $\mathrm{X}$ & $\mathrm{Y}$ & $\mathrm{Z}$ & $\mathrm{X}$ & Y & $\mathrm{Z}$ & $\mathrm{X}$ & $\mathrm{Y}$ & $\mathrm{Z}$ \\
\hline 1 & -6.3 & 3.6 & -2.7 & -3.6 & 1.8 & -0.9 & -4.5 & 0.9 & 0.0 \\
\hline 2 & -9.0 & 3.6 & -2.7 & -7.2 & 0.9 & -3.6 & -6.3 & 1.8 & 1.8 \\
\hline
\end{tabular}

Table 4. Deformable Model Slices Containing the Landmarks, in the One Plate Virtual Compression Experiment

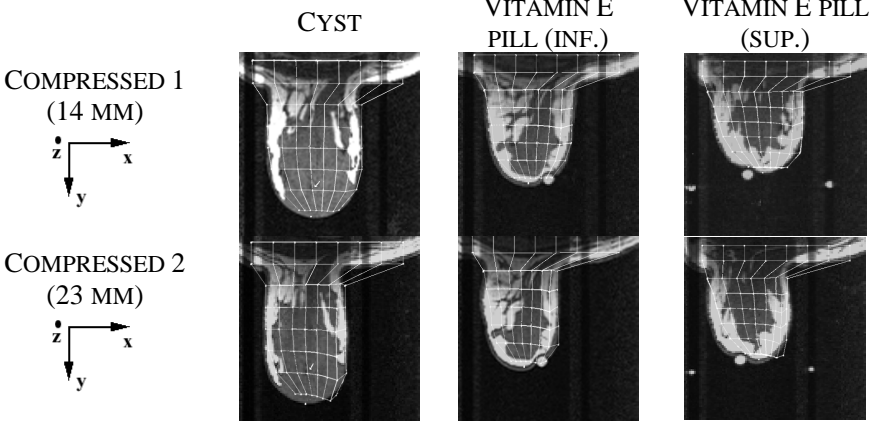

Table 5. Deformable Model Slices Containing the Landmarks, in the Two Plate Virtual Compression Experiment

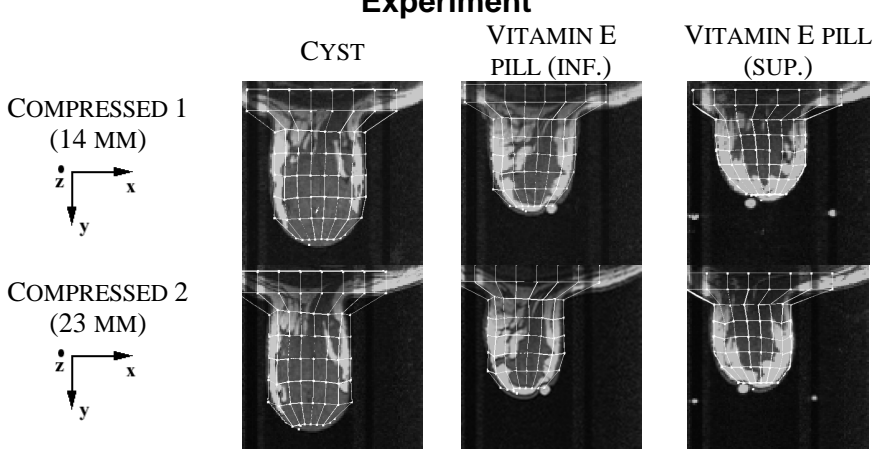

\section{Conclusion}

This initial breast compression study shows that it is possible to create a deformable model of the breast based on the use of finite elements with non-linear material properties capable of modeling the deformation of the breast. The geometry of the model is constructed from MR data, and the material properties of the different structures are computed independently using material testing techniques.

This deformable model will be used as a new tool to the physician, who will: 1) image the breast with no compression (thus increasing the contrast and visibility of the tumor), 2) use the compression plates (to minimize 
deformations caused by the insertion of the needle), 3) compress the breast model, and accurately locate the tumor within the real compressed breast during the procedure.

Table 6. Displacement Errors ( $\mathrm{mm})$ for the Three Landmarks and Average Boundary Fit Error (mm) (Modeled-Experimental)

\begin{tabular}{|c|c|c|c|c|c|c|}
\hline \multirow{3}{*}{$\begin{array}{l}\text { COMPRESSED } 1 \\
(14 \mathrm{MM})\end{array}$} & & & CYST & $\begin{array}{l}\text { PILL } \\
\text { (INF.) }\end{array}$ & $\begin{array}{l}\text { PILL } \\
\text { (SUP.) }\end{array}$ & $\begin{array}{c}\text { BOUNDARIES } \\
\text { FIT }\end{array}$ \\
\hline & (a) & $\begin{array}{l}\mathrm{X} \\
\mathrm{Y} \\
\mathrm{Z}\end{array}$ & $\begin{array}{c}1.1 \\
-1.9 \\
0.0\end{array}$ & $\begin{array}{c}1.7 \\
-0.6 \\
1.2\end{array}$ & $\begin{array}{l}-1.2 \\
2.1 \\
0.6\end{array}$ & 2.8 \\
\hline & (b) & $\begin{array}{l}\mathrm{X} \\
\mathrm{Y} \\
\mathrm{Z}\end{array}$ & $\begin{array}{c}-1.9 \\
-1.7 \\
0.4\end{array}$ & $\begin{array}{l}-1.1 \\
-1.7 \\
2.2\end{array}$ & $\begin{array}{l}-2.8 \\
2.6 \\
0.8\end{array}$ & 0.9 \\
\hline \multirow[t]{2}{*}{$\begin{array}{l}\text { COMPRESSED } 2 \\
(23 \mathrm{MM})\end{array}$} & (a) & $\begin{array}{l}\mathrm{X} \\
\mathrm{Y} \\
\mathrm{Z}\end{array}$ & $\begin{array}{c}1.2 \\
-2.0 \\
-0.9\end{array}$ & $\begin{array}{l}0.7 \\
2.7 \\
1.5\end{array}$ & $\begin{array}{l}3.0 \\
1.9 \\
2.0\end{array}$ & 4.5 \\
\hline & (b) & $\begin{array}{l}\mathrm{X} \\
\mathrm{Y} \\
\mathrm{Z}\end{array}$ & $\begin{array}{l}-4.4 \\
-1.9 \\
-1.3\end{array}$ & $\begin{array}{c}-1.2 \\
-1.9 \\
2.3\end{array}$ & $\begin{array}{r}-3.5 \\
1.0 \\
3.0\end{array}$ & 0.5 \\
\hline
\end{tabular}

(a) IS FOR ONE-PLATE VIRTUAL COMPRESSION, (b) IS FOR TWOPLATE VIRTUAL COMPRESSION.

\section{References}

1. Heywang SH, Wolf A, Pruss E, et al., "MR imaging of the breast with Gd-DTPA: use and limitations". Radiology, 171: 95-103 (1989)

2. Azar FS, Metaxas D, Schnall MD, "A finite element model of the breast for predicting mechanical deformations during interventional procedures". Proc. Intl. Soc. Magn. Reson. Med. 7: 1084 (1999).

3. Egan RL, "Breast Embryology, Anatomy and Physiology". Breast Imaging: Diagnosis and Morphology of Breast Diseases(Chap. 4): 30-58 (1988).

4. Egan RL, "Malignant breast lesions". Breast imaging: diagnosis and morphology of breast diseases(chap. 14): 227231 (1988).

5. $\quad$ "Understanding Breast Cancer Treatment". National Cancer Institute, NIH 98-4251: 6-7 (1998).

6. Speeter TH, "Three Dimensional Finite Element Analysis of Elastic Continua for Tactile Sensing". Intl Journal of Robotics Research, 11 No 1: 1-19 (1992).

7. Chen DT, Zeltzer D, "Pump it up: Computer animation of a biomechanically based model of the muscle using the finite element method". Computer Graphics (SIGGRAPH'92), 26: 89-98 (1992).

8. Reddy NP, Song GJ,, "Tissue cutting in virtual environments". Medicine meets virtual reality IV(IOS Press): 359-364 (1995).

9. Zienkiewicz OC, "The finite element method". McGraw-Hill, London, 3rd edition (1977).

10. Norton A, Turk G, Bacon B, Gerth J, Sweeney P, "Animation of fracture by physical modeling". The visual computer, 7 : 210-219 (1991).

11. Luciani A, Jimenez S, Florens JL, Cadoz C, Raoult O, "Computational physics: a modeler simulator for animated physical objects". Eurographics Workshop on Animation and Simulation: 425-437 (1991)

12. Joukhadar A, "Energy based adaptive time step and inertiamatrix based adaptive discretization for fast converging dynamic simulation". Proc. of the Intl. Workshop on Visualisation and Mathematics (1995).

13. Desbrun M, Gascuel MP, "Animating soft substances with implicit surfaces". Computer graphics (SIGGRAPH'95): $287-$ 290 (1995).
14. Baumann R, Glauser D, "Force feedback for virtual reality based minimally invasive surgery simulator". Medicine meets virtual reality, San Diego CA (1996).

15. Meseure P, Chaillou C, "Deformable body simulation with adaptive subdivision and cuttings". Proceedings of the WSCG'97: 361-370 (1997).

16. Kuehnapfel UG, Neisius B, "CAD-based graphical computer simulation in endoscopic surgery". end. surg., 1: 181-184 (1993).

17. Williams C, Clymer B, Schmalbrock P, "Biomechanics of breast tissue: preliminary study of force-deformation relationship". Proc. Intl. Soc. Magn. Reson. Med. 7: 524 (1999).

18. Sciaretta J, Bishop J, Samani A, Plewes DB, "MR validation of soft tissue deformation as modeled by non-linear finite element analysis". Proc. Intl. Magn. Reson. Med. 7: 246 (1999).

19. Metaxas D, "Physics-based modeling of nonrigid objects for vision and graphics". Ph.D. thesis, Dept. of computer science, U. of Toronto (1992).

20. Metaxas D, Terzopoulos D, "Shape and nonrigid motion estimation through physics-based synthesis". IEEE Trans. Pettern analysis and Machine Intelligence, 15(6): 569-579 (1993).

21. Fung YC, A first course in continuum mechanics. 3rd ed. 1994, Englewood Cliffs, NJ: Prentice Hall.

22. Hayes WC, Keer LM, Hermann G, Mockros LF, "A mathematical analysis for indentation tests of articular cartilage". J. Biomechanics, 5: 541-551 (1972).

23. Krouskop TA, Wheeler TM, Kallel F, Garra BS, Hall T, "The elastic moduli of breast and prostate tissues under compression". Ultrasonic imaging, 20: 151-159 (1998).

24. Sarvazyan AP, Skovoroda AR, Emelianov SY, Fowlkes JB, Pipi JG, Adler RS, Buxton RB, Carson PL, Biophysical bases of elasticity imaging. Acoustical imaging, ed. E.J. Jones. Vol. 21. 1995, New-York: Plenum Press.

25. Skovoroda AR, K.A., Gusakyan DA, Mayevskii YI, Yermilova VD, Oranskaya GA, Sarvazyan AP, "Quantitative analysis of the mechanical characteristics of pathologically changed soft biological tissues". Biophysics, 40(6): 13591364 (1995).

26. Fung YC, Biomechanics: mechanical properties of living tissues, 2nd edition. 1993, New-York: Springer Verlag.

27. Zhang M, Zheng YP, Mak AF, "Estimating the effective Young's modulus of soft tissues from indentation tests nonlinear finite element analysis of effects of friction and large deformation". Med. Eng. Phys., 19(6): 512-517 (1997).

28. Fung YC, "Biomechanics: mechanical properties of living tissues". New-York: Springer-Verlag: 203-212 (1981).

29. Spencer AJM, "Continuum mechanics". London, England: Longman: 153-163 (1980).

30. Haber I, Metaxas DN, Axel L, "Three-dimensional motion reconstruction and analysis of the right ventricle using tagged MRI". U. of Penn tech. report(MS-CIS-99-12) (1999).

31. Press WH, Teukolsky SA, Vetterling WT, Flannery BP, "Numerical recipes in C: the art of scientific computing". Cambridge University Press, Cambridge: 707-725 (1992).

32. DeVries PL, "A first course in computational physics". John Wiley \& Sons: 207-225 (1994).

33. Zienkiewicz OC, Taylor RL, "The finite element method". McGraw-Hill, New-York(fourth edition) (1989).

34. Lawrence AJ, Rossman PJ, Mahowald JL, Manduca A, Hartmann LC, Ehman RL, "Assessment of breast cancer by magnetic resonance elastography". Proc. Intl. Soc. Magn. Reson. Med. 7: 525 (1999).

35. Wellman PS, Howe RD, harvard Bio-Robotics Lab.Tech. Report, \#98-121 (1998).

36. Wellman PS, "Tactile Imaging". Ph.D. Thesis, Harvard University (1999). 